\title{
Inversion Methods for the Measurements of MHD- like Density Fluctuations by Heavy Ion Beam Diagnostic
}

\author{
A Malaquias', R B Henriques, I S Nedzelsky \\ Instituto de Plasmas e Fusão Nuclear, Instituto Superior Técnico, Universidade de Lisboa, \\ Av. Rovisco Pais, 1049-001 Lisboa, Portugal \\ E-mail: artur.malaquiaseipfn.ist.utl.pt
}

We report here on the recent developments in the deconvolution of the path integral effects for the study of MHD pressure-like fluctuations measured by Heavy Ion Beam Diagnostic. In particular, we develop improved methods to account for and remove the path integral effect on the determination of the ionization generation factors, including the double ionization of the primary beam. We test the method using the HIBD simulation code which computes the real beam trajectories and attenuations due to electron impact ionization for any selected synthetic profiles of plasma current, plasma potencial, electron temperature and density. Simulations have shown the numerical method to be highly effective in ISTTOK within an overall accuracy of a few percent $(<3 \%)$. The method here presented can effectively reduce the path integral effects and may serve as the basis to develop improved retrieving techniques for plasma devices working even in higher density ranges. As a test example an $m=1(100 \mathrm{kHz}) \mathrm{MHD}$ mode spatial profile evolution is retrieved with excellent accuracy after the reduction of path integral effects. The 2D MHD mode-like structure is reconstructed by means of a spatial projection of all 1D measurements obtained during one full rotation of the mode.

First EPs Conference on Plasma Diagnostics - $1^{\text {st }}$ ECPD

14-17 April 2015,

Villa Mondragone, Frascati (Rome) Italy

${ }^{1}$ Presenter

(c) Copyright owned by the author(s) under the terms of the Creative Commons Attribution-NonCommercial-ShareAlike Licence. 


\section{Introduction}

The efficient operation of a fusion reactor requires the active control of several plasma parameters and their spatial profiles within challenging accuracies. For instance, the ITER diagnostic system [1] comprising more than 40 sophisticated diagnostic techniques dedicated to machine protection, safe operation, plasma profiles control and physics studies will be used to ultimately provide the measurements necessary to develop the physics basis for operation of a DEMO grade device. As the demands on experimental measurements' resolution and accuracy increase in order to better understand plasma intrinsic physics processes and interplays the increasing size and power of the devices implies that the capability to diagnose the burning plasma becomes more limited due to the inherent constrains on access (e.g. shielding, complex interfacing of sub-systems, etc.) and on the operational requirements of fusion power plants (e.g. power loads, radiation levels, etc.). There is therefore a clear motivation nowadays to obtain the most complete plasma physics knowledge utilizing the existing small and medium size devices in view of their installed diagnostic capability and higher flexibility to conduct a broad range of experiments. In this regard the Heavy Ion Beam Diagnostic (HIBD) is a powerful tool that can in principle provide the local measurement of the quantities of interest being these the electron density and temperature, the internal poloidal field and the plasma potential with spatial resolution of several $\mathrm{mm}$ and down to the micro second range temporal resolution. For instance, experiments in ISTTOK [2][3][4][5], TJ-II [6], T-10 [6] and in the LHD device [7] have all together demonstrated the capabilities of the HIBD in plasma density, potential fluctuations and mean potential measurements, supporting MHD and turbulence analysis including Alfvèn Eigenmodes (TJ-II) [8] and Geodesic Acoustic modes (T-10) [9] studies.

Recently, the measurement capability in the ISTTOK HIBD has been extended to cover the full radial MHD fluctuation spectra up to $250-300 \mathrm{kHz}$ with phase preservation [10]. The signature of MHD activity is obtained from the detector cell currents fluctuations which relate to the plasma 'pressure-like' fluctuations via the product of local density and ionization cross section (a function of electron temperature). We present and test a retrieving method for removing the path integral effects in the measurements and reduce thus the presence of artifacts in the interpretation of the data in ISTTOK. Finally we propose and test a reconstruction method for the 2D profile of the MHD mode spatial structure.

\section{Trajectories and attenuations}

The HIBD is based on the injection of a single ionized beam (e.g. $\mathrm{Cs}^{+}, \mathrm{Hg}^{+}, \mathrm{Xe}^{+}$) into the plasma which will undergo impact ionization with the plasma electrons generating double charged ions (secondary beams). The ions' trajectories will be determined by the beam energy and the total magnetic fields (toroidal and poloidal) being the toroidal field the main driver for the final geometric definition of the HIBD [2], establishing a unique correspondence between each detector cell and the sample volume in the plasma (Fig. 1a). The detector currents are determined by the effective ionization cross sections (a function of local temperature) and local plasma density. In the case of ISTTOK tokamak ( $\left.r=0.085 \mathrm{~m}, T_{e} \sim 100-150 \mathrm{eV}, n_{e} \sim 6.5 \mathrm{e} 18 \mathrm{~m}-3\right)$ the relevant reactions contributing to the detected currents are i) the single and double ionization of the primary beam and ii) the single ionization of the secondary beams. The equation describing such ionization processes leading to the detected currents is given by 

Diagnostic

$$
\begin{aligned}
& \mathrm{I}_{j(\mathrm{det})}^{2+}=2 \mathrm{I}_{0}^{+} \underbrace{\exp \left(-\int_{R_{i}}^{r_{j}} n_{e}\left(s_{1}\right)\left(\hat{\sigma}_{12}+\hat{\sigma}_{13}\right) d s_{1}\right)}_{A} \\
& . n_{e}\left(r_{j}\right) \hat{\sigma}_{12}\left(r_{j}\right) d l_{(\text {cell })} \cdot \underbrace{\exp \left(-\int_{r_{j}}^{R p} n_{e}\left(s_{2}\right) \hat{\sigma}_{23} d s_{2}\right)}_{B}
\end{aligned}
$$

In eq. $1 I_{0}^{+}$is the injected primary beam current, $n\left(s_{i}\right)$ is the local plasma electron density evaluated over the beam path ( $\mathrm{i}=1$ for primary beam and $\mathrm{i}=2$ for secondary beam), $\hat{\sigma}_{12}$ and $\hat{\sigma}_{13}$ are respectively the temperature dependent effective cross sections for the single and double ionization of the primary beam, and $\hat{\sigma}_{23}$ is the effective cross section for single ionization of the secondary beam. The factor 2 accounts for the double charge of the detected secondary ions. The factor $\mathrm{A}$ is the integral of attenuation of primary beam from the plasma entrance $\left(R_{i}\right)$ to the ionization point $\left(r_{j}\right)$. Likewise, the factor B accounts for the attenuation of the secondary beam from the ionization point $\left(r_{j}\right)$ until the exit of the plasma $\left(R_{p}\right)$. The production of secondaries at each sample volume is governed by the local generation factor $n_{e}\left(r_{j}\right) \hat{\sigma}_{12}\left(r_{j}\right)$, which is the indirect measured quantity from the detector currents. The size of the detector cell projected along the primary beam trajectory is $d l$ (in eq.1). This work extends the retrieving method presented in the reference [3] to include the attenuation of primary beam via double ionization (we strongly suggest the reader to get familiar with the method there presented). This addition is necessary in order to recover the local generation factor absolute values $n_{e}\left(r_{j}\right) \hat{\sigma}_{12}\left(r_{j}\right)$ with higher accuracy.

\subsection{Attenuation of primary beam due to double ionization}

The difference between the initial injected current $I_{0}^{+}$and the collected $I_{d e t}^{+}$current of the primary beam corresponds to the current lost in the generation of secondary ions $\left(I_{\text {total }}^{2+}\right)$ and tertiary ions $\left({ }^{1 \rightarrow 3} I_{\text {total }}^{3+}\right)$,

$$
I_{0}^{+}-I_{\mathrm{det}}^{+}=\frac{1}{2} I_{\text {total }}^{2+}+\frac{1}{3}\left(1 \rightarrow 3 I_{\text {total }}^{3+}\right)
$$

The ratio between the right-hand-side quantities namely, the total current of tertiary ions, $1 \rightarrow 3 I_{\text {total }}^{3+}$, and total current of secondary ions, $I_{\text {total }}^{2+}$ (both generated by the primary beam), was evaluated for a range of temperatures and density profiles using the HIBD simulation code.



Fig. 1a - Sample volumes along the primary beam trajectory. The circle delimits the maximum plasma radius

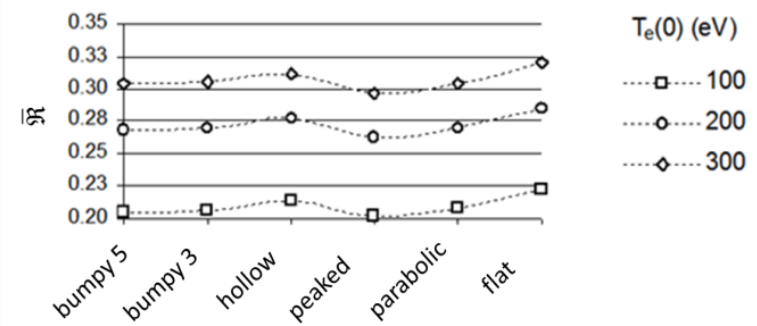

Fig. 1b - Ratio between tertiary and secondary ion currents (from the primary beam) for different profiles and central temperatures. 
We found that for a fixed central temperature this ratio does not depend strongly on the temperature profiles and is independent of the density profile (because both generated currents secondary and tertiary arise from the same sampling volume). As shown in Fig. 1b this ratio is in a good approximation mainly determined by the value of plasma central temperature. This result allows to relate these two quantities by a factor $\overline{\mathfrak{R}}$ (profile averaged) which depends mainly on the plasma central temperature $\left(T_{e}(0)\right.$ is required to be known experimentally or equivalently the average temperature via Spitzer resistivity),

$$
\frac{\left(1 \rightarrow 3 I_{\text {total }}^{3+}\right)}{I_{\text {total }}^{2+}}=\overline{\mathfrak{R}}
$$

From eq. 3 one obtains ${ }^{1 \rightarrow 3} I_{\text {total }}^{3+}$ and replacing in eq. 2 the value of $I_{\text {total }}^{2+}$ is found (a $2 / 3$ charge correction factor must be used when replacing in eq. 2 as to conserve the total number of ions). Reference [3] describes the calculation of the current lost by the primary beam to secondary ions for each ionization volume based on the value of $I_{\text {total }}^{2+}$. The following section describes the estimation of the current lost by the primary beam to tertiary ions for each sample volume based on the value of ${ }^{1 \rightarrow 3} I_{\text {total }}^{3+}$.

\subsection{Retrieval of the absolute values of the local generation factor $n_{e}\left(r_{j}\right) \hat{\sigma}_{12}\left(r_{j}\right)$}

For the estimation of the tertiary current generated at each ionization point along the primary beam ${ }^{1 \rightarrow 3} I_{j}^{3+}$ one can use a corresponding normalized weighing function given by,

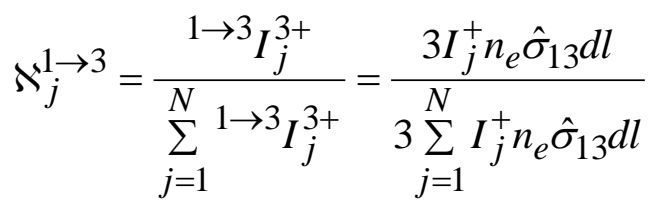

The numerator represents the current lost by the primary beam in the generation of a single tertiary beam and the denominator is the sum of all individual tertiary beam currents. In this equation we do not know the shape of $n \hat{\sigma}_{13}$. Since at this point we are interested mostly in the profiles rather than in absolute values we make use of the quasi-matching shapes between $n \hat{\sigma}_{12}$ and $\mathrm{n} \hat{\sigma}_{13}$ generation factors (as confirmed by calculations for a wide variety of profiles for $n_{e}$ and $T_{e}$ ). The profile of $\mathrm{n} \hat{\sigma}_{12}$ is obtained by the relative method described in [3] and can be used to replace $n \hat{\sigma}_{13}$ in eq. (4),

$$
\aleph_{j}^{1 \rightarrow 3}=\frac{I_{j}^{+} n_{e} \hat{\sigma}_{12} d l}{\sum_{j=1}^{N} I_{j}^{+} n_{e} \hat{\sigma}_{12} d l}=\frac{I_{j}^{2+}}{\sum_{j=1}^{N} I_{j}^{2+}} \cong \frac{I_{j(\mathrm{det})}^{2+}}{\sum_{j=1}^{N} I_{j(\mathrm{det})}^{2+}}
$$

where the last equality is justified for ISTTOK plasma by the fact that the relative distribution of secondary currents in the detector cells follows the relative distribution of the secondary 
currents at the ionization point [3]. Finally we account for the local double ionization of the primary beam via the product: ${ }^{1 \rightarrow 3} I_{j}^{3+}=3 . \aleph_{j}^{1 \rightarrow 3} \cdot 1 \rightarrow 3 I_{\text {total }}^{3+}$. The set of equations here developed was used to extend the method in reference [3]. The local generation factor $n_{e}\left(r_{j}\right) \hat{\sigma}_{12}\left(r_{j}\right)$ can thus be obtained with an accuracy $<3 \%$ of the absolute values for a wide range of profiles (the same profiles as used to produce the results in Fig. 1).

\section{Retrieving of MHD $n_{e}\left(r_{j}\right) \hat{\sigma}_{12}\left(r_{j}\right)$ pressure-like fluctuations}

The above method can retrieve the local $n_{e}\left(r_{j}\right) \hat{\sigma}_{12}\left(r_{j}\right)$ values. This product we call it 'pressure-like' because of its dependence via the effective ionization cross section with plasma temperature for the range of ISTTOK plasmas $(30-130 \mathrm{eV})$. The method was tested for retrieving a $\mathrm{m}=1$ mode located at $\mathrm{r}=0.025 \mathrm{~m}$. In Fig. $2 \mathrm{a}$ is presented the mode structure (electron density and temperature) as inputted in the simulation code. The mode rotates with a $100 \mathrm{kHz}$ frequency and the acquisition system samples at a rate of $2 \mathrm{MHz}$. In Fig. 2b, is depicted the time evolution of the inputted $n_{e}\left(r_{j}\right) \hat{\sigma}_{12}\left(r_{j}\right)$ values for each ionization volume overlapped with the retrieved $n_{e}\left(r_{j}\right) \hat{\sigma}_{12}\left(r_{j}\right)$ calculated using the above method. As it can be seen the average accuracy is of a very few percent.

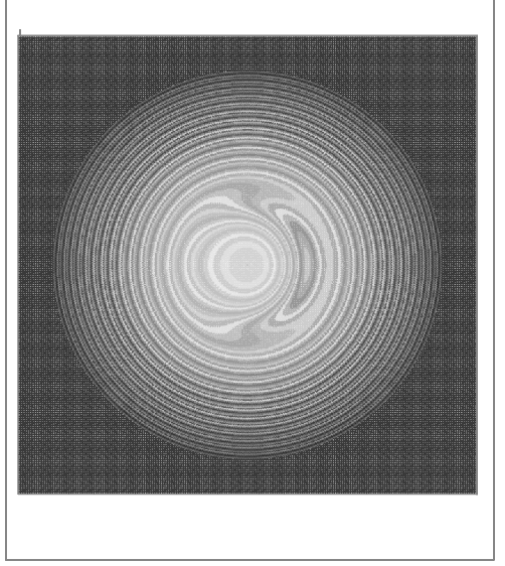

Fig 2.a $-n_{e}\left(\right.$ or $T_{e}$ ) profile (the 2D dimension is $0.1 \times 0.1 \mathrm{~m}$ )

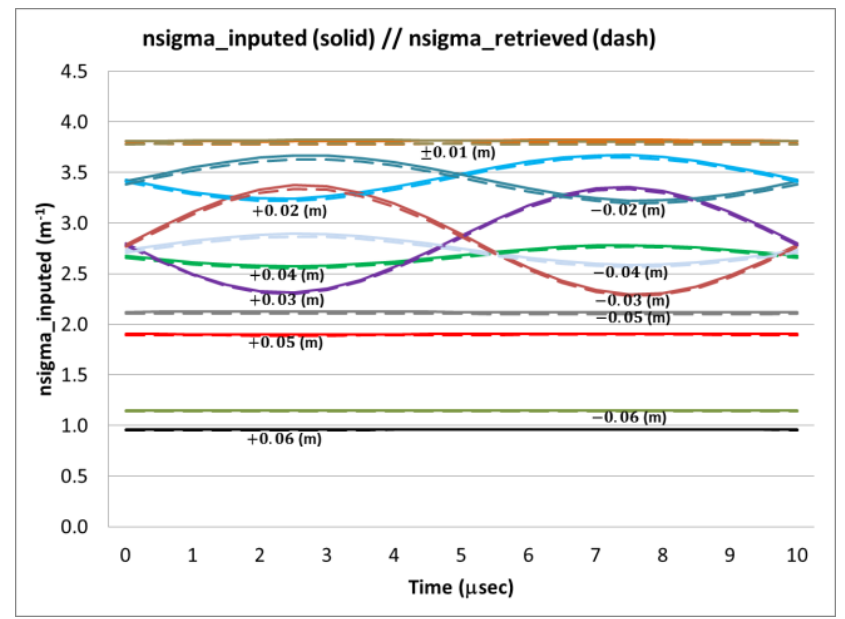

Fig 2.b - Evolution of local $n_{e}\left(r_{j}\right) \hat{\sigma}_{12}\left(r_{j}\right)$ values: inputted (full line) and retrieved (broken line) at each sub-labeled radius.

\section{MHD mode reconstruction}

The beam time-of-flight in the plasma $(\sim 0.6 \mu \mathrm{s})$ is much shorter than the fluctuations rate in the plasma $(\mathrm{kHz})$. Therefore, one can consider the HIBD measurements instantaneous photographs taken at each sampling interval $(0.5 \mu \mathrm{s})$. Knowing the mode number (from Mirnov coils) and frequency (either from Mirnov coils or HIBD) one can take several 1D snapshots of $n_{e} \hat{\sigma}_{12}\left(r_{j}\right)$ during a full rotation of the mode and project them in the 2D coordinated system of the chamber. The method is schematically depicted in Fig 3a. For a given mode rotation frequency, $F_{M}$, the corresponding number of synchronized snapshots is given by the ratio $N_{F}=F_{S} / F_{M}$ where $F_{S}$ is the sampling frequency. The angle between each snapshot is given by $\theta_{S}=2 \pi / \mathrm{N}_{\mathrm{F}}$. For each 
particular angle $\Sigma \theta_{\mathrm{S}}$ we can calculate the rotated $\mathrm{x}$ and $\mathrm{y}$ coordinate of each of the twelve sample volumes and take the value of $n_{e} \hat{\sigma}_{12}\left(r_{j}\right)$ at that position:

$$
n_{e} \hat{\sigma}_{12}\left(x_{j}, y_{j}\right)_{\Sigma \theta_{\mathrm{S}}} \text { with } x_{j}=r_{j} \sin \left(\Sigma \theta_{S}\right) ; y_{j}=r_{j} \cos \left(\Sigma \theta_{S}\right)
$$

The calculation stops when the cumulative angle $\Sigma \theta_{\mathrm{S}}=2 \pi$. The result is shown in Fig. 3b which presents the retrieved absolute values of $n_{e} \hat{\sigma}_{12}\left(x_{j}, y_{j}\right)_{\Sigma \theta_{\mathrm{S}}}$ for one full rotation of the mode.



Fig. 3a - Equivalent coordinates of the 1D rotating axis system (beam) scanning the mode (considered stationary).

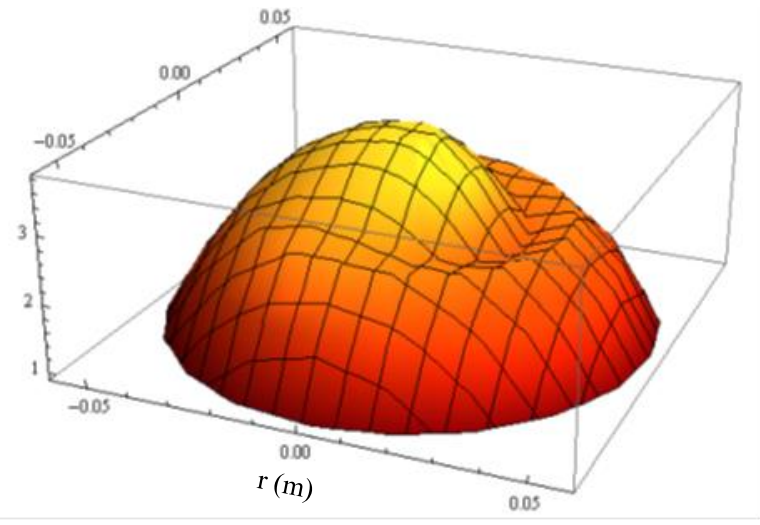

Fig. $3 \mathrm{~b}-2 \mathrm{D}$ reconstruction of the $n_{e} \hat{\sigma}_{12}\left(r_{j}\right)$ profile by $1 \mathrm{D}$ spatial projection through the full mode rotation. Here the top half diameter of the primary beam path was used for the reconstruction.

\section{Conclusions}

A retrieving algorithm was developed taking into account the double ionization of the primary beam resolving the local values of the $n_{e} \hat{\sigma}_{12}\left(r_{j}\right)$ generation factor within a $3 \%$ accuracy. This method effectively removes path integral effects that are responsible to produce artifacts in the spectral composition of the detected signals and in the cross correlation analysis. A method to reconstruct the $2 \mathrm{D}$ mode profile was presented based on the spatial projection of all measurements taken during one rotation of the mode.

\section{References}

[1] Nucl. Fusion 47 (2007) S337-S384 doi:10.1088/0029-5515/47/6/S07

[2] A. Malaquias, et al., Fusion Engineering and Design, 34-35 (1997) 671-674

[3] A. Malaquias, et al., Rev. of Sci. Instruments, V70, N1, Jan 1999, Part II. Pp. 947-950

[4] I.S.Nedzelskiy, et al., Rev. Sci. Instruments, 72, 1, 572-574, 2001

[5] R. B. Henriques, et al., Rev. Sci. Instrum. 83, 10D705 (2012)

[6] A. V. Melnikov, et al., Nucl. Fusion 51 (2011) 083043

[7] T. Ido et al., Rev. Sci. Inst. 2008 Oct 79 (10): 10F318

[8] A.V. Melnikov et al., Nucl. Fusion 50 (2010) 084023

[9] A.V. Melnikov et al., Plasma Phys. Control. Fusion 48 No 4 (April 2006) S87-S110

[10] R B Henriques et al., Review Of Scientific Instruments 85, $11 \mathrm{~d} 848$ (2014)

Acknowledgments:

This work was supported by the project UID/FIS/50010/2013 from "Fundação para a Ciência e a Tecnologia" 